\title{
Concurrent training and Eri silkworm pupae ingestion improve resting and exercise fat oxidation and energy expenditure in obese adults
}

\author{
Andaman Klomklorm', Ratree Ruangthai', Pilanee Vaithanomsat'², Udomlak Sukatta², Jatuporn Phoemsapthawee 1,* \\ 'Department of Sports Science and Health, Faculty of Sports Science, Kasetsart University, Nakhon Pathom, Thailand \\ ${ }^{2}$ Kasetsart Agricultural and Agro-Industrial Product Improvement Institute, Kasetsart University, Bangkok, Thailand
}

A randomized control trial was conducted to investigate the effects of combined concurrent training and Eri silkworm pupae ingestion on resting and exercise fat oxidation (FAO), as well as energy expenditure, and cardiometabolic risk markers in obese adults. Thirty-six sedentary, obese participants were divided into three groups: (1) placebo control group (CON, $n=12$ ), (2) Eri silkworm pupae ingestion group (ERI, $n=12$ ), and (3) combined concurrent training and Eri silkworm pupae ingestion group (CBT-ERI, $n=12$ ). Participants in the ERI and the CBT-ERI consumed 5-g Eri silkworm pupae/day (approximately 2.5-g linolenic acid). The concurrent training program comprised of supervised aerobic and resistance training: three 1-hr sessions/wk for 8 weeks. Body composition, energy expenditure, and FAO at rest and during exercise, heart rate variability, and blood chemistry were measured before and after the 8-week interventions. Following the interventions, resting FAO, the natural logarithm of very low-frequency power (InVLF), and high-sensi- tive C-reactive protein concentration significantly improved in both the ERI and the CBT-ERI. Only the CBT-ERI improved resting energy expenditure, FAO during exercise, trunk and gynoid fat mass, total cholesterol concentration, the standard deviation of normal R-R intervals (SDNN), and the percentage difference between adjacent normal R-R intervals $>50 \mathrm{~ms}$. Furthermore, there were significant correlations between resting energy expenditure and FAO, InVLF and SDNN in the CBT-ERI. In conclusion, this study demonstrates that concurrent training together with dietary Eri silkworm pupae leads to increased energy expenditure through a significant increase in $\mathrm{FAO}$ at rest and during exercise, as well as reduced fat mass.

Keywords: Concurrent training, Eri silkworm pupae, Energy expenditure, Fat oxidation, Obesity, Polyunsaturated fatty acid

\section{INTRODUCTION}

Obese individuals display an impaired capacity to mobilize and oxidize fat compared to lean individuals. This impaired capacity contributes to positive fat balance resulting in adipose tissue accumulation, and eventually weight gain (Blaak and Saris, 2002). Obesity relates directly to risk of cardiovascular diseases (CVDs). Therefore, preventing weight gain and facilitating weight loss are cornerstones to reducing the risk of CVD (Lavie et al., 2009).

Regular exercise is a key component of clinical weight management due to its ability to increase energy expenditure and promote fat utilization (Goodpaster et al., 2003; Potteiger et al., 2008;

Ryan and Heaner, 2014). Nevertheless, the influence of exercise training on fat oxidation (FAO) and energy expenditure in overweight and obese individuals is still unclear. There is a lack of consistent results detailing exercise training promotion of FAO in obese individuals. Some reports demonstrated the improvement of FAO caused by exercise training (Schrauwen et al., 2002; Schutz et al., 1992), while some studies reported contradictory results (Buemann et al., 1992; Nicklas et al., 1997). Similarly, the effect of exercise training on resting energy expenditure is in doubt.

Aerobic exercise has been widely accepted amid reductions in body weight (BW) and fat mass (FM) (Potteiger et al., 2008). How-

${ }^{*}$ Corresponding author: Jatuporn Phoemsapthawee

(iD) https://orcid.org/0000-0001-8859-3899

Department of Sports Science and Health, Faculty of Sports Science, Kasetsart University 73140, Thailand

E-mail: jatuporn.w@ku.th

Received: September 18, 2020 / Accepted: October 4, 2020 
ever, the majority of aerobic exercise studies failed to demonstrate increments in fat-free mass and resting energy expenditure (Wilmore et al., 1999). Moreover, loss of muscular tissue is a common problem often found in weight management programs. Adding resistance training into an aerobic exercise program is a strategy to prevent loss of muscle mass. Additionally, resistance training promotes energy expenditure and $\mathrm{FAO}$ which assist to maintain energy balance and prevent weight gain (Kirk et al., 2009).

Although the benefits of aerobic exercise and resistance training are well documented, little is known regarding the effects of combined aerobic exercise and resistance training, i.e., concurrent training in obese individuals. A meta-analysis in obese adults suggested that concurrent training is the most efficacious means to improve adiposity parameters as compared with aerobic exercise or resistance training alone (Schwingshackl et al., 2013). Moreover, a recent randomized controlled trial suggested that concurrent training produces greater decreases in body fat percentage $(\% \mathrm{BF})$, waist circumference, and body mass index (BMI) than aerobic exercise alone (Sigal et al., 2014).

Much attention has focused on the influence of dietary fat composition in energy and weight balance (Krishnan and Cooper, 2014; Piers et al., 2002; Polley et al., 2018; Stevenson et al., 2017). Numerous studies demonstrated the benefits of dietary polyunsaturated fatty acids (PUFAs) (Saini and Keum, 2018). Consuming PUFAs has been shown to elicit moderate body fat-lowering effects (Albracht-Schulte et al., 2018; Stevenson et al., 2017). High PUFAs consumption may potentially lead to improved weight maintenance and positive effects amid energy expenditure due to increments in thermogenesis, $\mathrm{FAO}$ and resting energy expenditure (Jones et al., 2008). High PUFAs consumption also provides beneficial effects in terms of insulin sensitivity, anti-inflammation, and blood lipid lowering (Albracht-Schulte et al., 2018).

Currently, in Thailand, Eri silkworm rearing is being widely promoted in public and private sectors. Pupae, which accounts for $60 \%$ of the cocoon weight, is discarded as waste material. However, the Eri silkworm pupae were found to be enriched with PUFAs such as palmitic acid, oleic acid, and linoleic acid (Kim et al., 2010) as well as $\alpha$-linolenic acid (Shanker et al., 2006). A nutritional and toxicological study on the Eri silkworm pupae oil showed that it is safe and nutritionally equivalent to commonly used sun-flower oils, but with added health benefits due to its high $\alpha$-linolenic acid content (Longvah et al., 2012). Moreover, a comprehensive review of their toxicological assessment has indicated that the Eri silkworm pupae is safe and has great potential as novel food resources (Gao et al., 2018). Therefore, promoting the pupae as an alterna- tive food would provide added value to the discarded silkworm.

As mentioned above, concurrent training as well as dietary PUFAs consumption yields beneficial effects on weight management. Nevertheless, the combined effects of concurrent training together with PUFAs intake have not yet been studied. Thus, we hypothesized that the combination of concurrent training with Eri PUFAs intake would improve energy expenditure and FAO at rest and during submaximal exercise, as well as cardiometabolic risk markers in obese adults.

\section{MATERIALS AND METHODS}

\section{Participants}

A total of 36 middle-aged ( $41.6 \pm 8.3$ years) overweight or obese (BMI, $28.3 \pm 3.9 \mathrm{~kg} / \mathrm{m}^{2} ; \% \mathrm{BF}, 41.1 \% \pm 5 \%$ ) adults (33 women and 3 men) were recruited for this study. Of these, $58.3 \%$ and $27.8 \%$ had hypercholesterolemia and hypertriglyceridemia, respectively. All participants did not engage in regular physical exercise ( $>1$ time/wk) and all had been at a stable weight $( \pm 3 \mathrm{~kg})$ for at least 6 months prior to the study. Participants were excluded if they had a history of type 2 diabetes, coronary heart disease, peripheral vascular disease, uncontrolled hypertension, or if they were taking chronic medications known to affect energy homeostasis. We also excluded participants who had amid screening, been observed for a clinical hypothyroidism, or elevated liver enzymes. The study protocol was conducted according to the Declaration of Helsinki and approved by the Kasetsart University Research Ethics Committee (COA61/084). Sample size was based on the ability to detect a large effect (1.3) according to a previous report by Solomon et al. (2008). As a consequence, it was decided upon to require $80 \%$ power at 0.05 significance. Thus, having at least 11 participants in each group was required to complete the study.

\section{Study design}

Randomized placebo-controlled trial was undertaken with participants blinded from test results to reduce experimental bias. After screening, all participants were matched for age, gender, BMI, and $\% \mathrm{BF}$ and randomly assigned into three groups: (1) a placebo control (CON, $\mathrm{n}=12,91.7 \%$ female), (2) an Eri silkworm pupae ingestion group (ERI, $\mathrm{n}=12,91.7 \%$ female), and (3) a combined concurrent training and Eri silkworm pupae ingestion group (CBT-ERI, $\mathrm{n}=12,91.7 \%$ female). Participants' characteristics are presented in Table 1.

The CON participants followed study procedures similarly to 
Table 1. Anthropometrics, body composition, and cardiometabolic risk markers at baseline and post interventions

\begin{tabular}{|c|c|c|c|c|c|c|}
\hline \multirow{2}{*}{ Variable } & \multicolumn{2}{|c|}{$\operatorname{CON}(n=12)$} & \multicolumn{2}{|c|}{$E R I(n=12)$} & \multicolumn{2}{|c|}{ CBT-ERI $(n=12)$} \\
\hline & Baseline & Posttest & Baseline & Posttest & Baseline & Posttest \\
\hline Age (yr) & $38.2 \pm 7.5$ & & $42 \pm 6.6$ & & $44.3 \pm 10.1$ & \\
\hline Body weight (kg) & $70.8 \pm 10.7$ & $70.5 \pm 11.1$ & $68.2 \pm 16.2$ & $68.3 \pm 16.3$ & $73.5 \pm 6.6$ & $71.7 \pm 6.3^{*}$ \\
\hline $\mathrm{BMI}\left(\mathrm{kg} / \mathrm{m}^{2}\right)$ & $28.2 \pm 3.1$ & $28 \pm 3.1$ & $27.9 \pm 5.5$ & $27.8 \pm 5.6$ & $28.8 \pm 3.0$ & $28 \pm 3.1^{* *}$ \\
\hline Body fat (\%) & $41.1 \pm 5.8$ & $41 \pm 6.1$ & $42 \pm 3.9$ & $42.2 \pm 3.8$ & $40.7 \pm 5.5$ & $39.4 \pm 6.2^{*}$ \\
\hline Fat mass (kg) & $28.3 \pm 7.5$ & $28.4 \pm 8.2$ & $28.1 \pm 9.3$ & $28.3 \pm 9.3$ & $29.2 \pm 4.4$ & $27.5 \pm 4.8^{* *}$ \\
\hline Fat free mass $(\mathrm{kg})$ & $42.1 \pm 4.8$ & $42.4 \pm 4.9$ & $40.2 \pm 7.4$ & $40.3 \pm 7.8$ & $45.0 \pm 6.5$ & $44.7 \pm 6.7$ \\
\hline SMM (kg) & $39.8 \pm 4.6$ & $40.1 \pm 4.7$ & $38.1 \pm 7.1$ & $38.1 \pm 7.5$ & $42.7 \pm 6.4$ & $42.4 \pm 6.5$ \\
\hline Visceral fat (kg) & $0.94 \pm 0.6$ & $0.95 \pm 0.6$ & $0.91 \pm 0.4$ & $0.92 \pm 0.4$ & $0.95 \pm 0.4$ & $0.94 \pm 0.4$ \\
\hline $\operatorname{VFA}\left(\mathrm{cm}^{3}\right)$ & $997.7 \pm 607.9$ & $1,007.5 \pm 657.3$ & $962.4 \pm 461.4$ & $975.4 \pm 461.3$ & $1,009.6 \pm 462.6$ & $1,000.8 \pm 450.7$ \\
\hline Arm fat (kg) & $3.4 \pm 1.0$ & $3.4 \pm 1.0$ & $3.5 \pm 1.1$ & $3.5 \pm 1.2$ & $3.4 \pm 0.6$ & $3.2 \pm 0.5^{*}$ \\
\hline Leg fat (kg) & $9.8 \pm 2.7$ & $9.5 \pm 2.8$ & $9.9 \pm 3.3$ & $9.8 \pm 3.3$ & $9.9 \pm 2.5$ & $9.5 \pm 2.6^{*}$ \\
\hline Trunk fat (kg) & $14.2 \pm 4.1$ & $14.6 \pm 4.7$ & $13.9 \pm 5.2$ & $14.1 \pm 5.2$ & $14.9 \pm 2.3$ & $14.0 \pm 3^{* \dagger, \pm}$ \\
\hline Android fat (kg) & $2.3 \pm 0.9$ & $2.3 \pm 1.0$ & $2.3 \pm 0.9$ & $2.3 \pm 1.0$ & $2.4 \pm 0.4$ & $2.3 \pm 0.5^{*}$ \\
\hline Gynoid fat (kg) & $4.8 \pm 1.3$ & $4.7 \pm 1.3$ & $4.8 \pm 1.5$ & $4.8 \pm 1.5$ & $4.9 \pm 1.0$ & $4.6 \pm 1.0^{*,+, \pm}$ \\
\hline $\mathrm{A} / \mathrm{G}$ ratio & $0.5 \pm 0.1$ & $0.5 \pm 0.1$ & $0.5 \pm 0.1$ & $0.5 \pm 0.1$ & $0.5 \pm 0.1$ & $0.5 \pm 0.1$ \\
\hline Waist circumference $(\mathrm{cm})$ & $85.8 \pm 10.8$ & $84.4 \pm 9.6$ & $86.9 \pm 10.4$ & $84.1 \pm 10.3^{*}$ & $91.6 \pm 5.4$ & $87 \pm 6.5^{* *}$ \\
\hline Hip circumference (cm) & $103.9 \pm 8.1$ & $103 \pm 6.8$ & $104.1 \pm 10.3$ & $101.8 \pm 10.5^{*}$ & $106.1 \pm 4.1$ & $103.8 \pm 4.4^{*}$ \\
\hline W/H ratio & $0.83 \pm 0.1$ & $0.82 \pm 0.1$ & $0.84 \pm 0.1$ & $0.83 \pm 0.1$ & $0.86 \pm 0.1$ & $0.84 \pm 0.1^{*}$ \\
\hline Fasting blood glucose (mg/dL) & $90.5 \pm 16.1$ & $101.1 \pm 17.6^{* *}$ & $105.2 \pm 51.9$ & $128.8 \pm 76.5^{* *}$ & $86.7 \pm 9.0$ & $89.3 \pm 8.7$ \\
\hline Insulin (IU/mL) & $12.7 \pm 3.9$ & $15.9 \pm 6.9^{*}$ & $10.2 \pm 4.1$ & $13.8 \pm 6.2^{*}$ & $11.0 \pm 5.1$ & $10.4 \pm 3.2$ \\
\hline HOMA-IR & $2.9 \pm 1.1$ & $4.1 \pm 2.3^{*}$ & $2.7 \pm 2.0$ & $4.2 \pm 2.5^{* *}$ & $2.4 \pm 1.1$ & $2.3 \pm 0.6$ \\
\hline hs-CRP (mg/L) & $3.8 \pm 3.9$ & $2.7 \pm 1.3$ & $6.7 \pm 7.9$ & $4.1 \pm 1.5^{*}$ & $2.0 \pm 0.8$ & $1.4 \pm 0.8^{*, \pm}$ \\
\hline Leptin (ng/mL) & $12.9 \pm 9.5$ & $15.2 \pm 7.6$ & $10.8 \pm 5.7$ & $12.9 \pm 6.2$ & $11.5 \pm 8.1$ & $10.4 \pm 6.7$ \\
\hline $\mathrm{TC}(\mathrm{mg} / \mathrm{dL})$ & $213.8 \pm 37.5$ & $226.2 \pm 57.4$ & $201.3 \pm 20.1$ & $220.5 \pm 76.5^{*}$ & $219.3 \pm 34.4$ & $195.3 \pm 32.6^{* *, t t, \text { t中 }}$ \\
\hline $\mathrm{TG}(\mathrm{mg} / \mathrm{dL})$ & $119.7 \pm 62.8$ & $134.4 \pm 87.9$ & $109.9 \pm 56.5$ & $152.2 \pm 93.0^{*}$ & $135.9 \pm 119.1$ & $135.6 \pm 74.4$ \\
\hline LDL-C (mg/dL) & $119.7 \pm 32.1$ & $135.3 \pm 35.0^{*}$ & $113.4 \pm 24.8$ & $130.8 \pm 34.0^{* *}$ & $115.9 \pm 27.2$ & $123.6 \pm 22.7$ \\
\hline $\mathrm{HDL}-\mathrm{C}(\mathrm{mg} / \mathrm{dL})$ & $62.1 \pm 8.1$ & $70.2 \pm 10.1$ & $62.1 \pm 14.6$ & $67.8 \pm 14.2$ & $61.1 \pm 12.2$ & $57.5 \pm 10.2$ \\
\hline TG/HDL-C & $2 \pm 1.2$ & $1.9 \pm 1.2$ & $2.0 \pm 1.3$ & $2.4 \pm 1.5$ & $2.5 \pm 2.6$ & $2.5 \pm 1.6$ \\
\hline LDL-C/HDL-C & $1.9 \pm 0.6$ & $1.9 \pm 0.5$ & $2.0 \pm 0.8$ & $2.1 \pm 0.9$ & $2.0 \pm 0.7$ & $2.2 \pm 0.6$ \\
\hline
\end{tabular}

Values are presented as mean \pm standard deviation.

CON, control group; ERI, Eri silkworm pupae ingestion; CBT-ERI, combined concurrent training and Eri silkworm pupae ingestion; BMI, body mass index; SMM, skeletal muscle mass; VFA, visceral fat area; A/G ratio, android fat to gynoid fat ratio; W/H ratio, waist-to-hip ratio; HOMA-IR, homeostatic model assessment of insulin resistance; hs-CRP, high-sensitive C-reactive protein; TC, total cholesterol; TG, triglyceride; LDL-C, low-density lipoprotein cholesterol; HDL-C, high-density lipoprotein cholesterol.

${ }^{*} P<0.05$ and ${ }^{* *} P<0.01$ to within-group comparison (baseline vs. post interventions). ${ }^{\dagger} P<0.05$ and ${ }^{t+} P<0.01$ to between-group comparison (vs. CON). ${ }^{\ddagger} P<0.05$ and ${ }^{\ddagger \ddagger} P<0.01$ to between-group comparison (vs. ERI).

those in the intervention groups to ensure they did not know they were in the CON. All groups were asked to consume a snack bar twice a day. The snack bars were the same shape, color, and odor. The snack bars in the CON contained no Eri silkworm pupae, while the snack bars in the others contained $5 \mathrm{~g}$ of Eri silkworm pupae per day (approximately $2.5 \mathrm{~g}$ of linolenic acid). In addition, only the CBT-ERI participants performed 60-min of concurrent training 3 days per week. All participants were asked to maintain their habitual activity levels besides the interventions throughout the experimental period. They were requested to maintain food intake i.e., the same intake levels as preceding study outset. Nutrition logs were monitored to keep track of subjects' dietary intake. Participants completed baseline measurements over one week before attending an initiation supervised exercise session at the Sports Science fitness center. Measurements were conducted at the cardiometabolic laboratory at the Faculty of Sports Science, Kasetsart University for blood sample collection, anthropometrics, body composition, FAO at rest and during exercise as well as energy expenditure, and heart rate variability (HRV) at baseline, and following the 8-week interventions. 
Table 2. Nutrition facts and total fatty acid percentage in Eri snack bar

\begin{tabular}{lc}
\hline Variable & Value \\
\hline Total fat (g) & 5 \\
Saturated fat (g) & 2 \\
Saturated fatty acid (\%) & \\
Steric acid & 4.7 \\
Arachidic acid & 0.7 \\
Polyunsaturated fatty (g) & 3 \\
Polyunsaturated fatty acid (\%) & \\
Palmitoleic acid & 0.8 \\
Oleic acid & 11.3 \\
Linoleic acid & 5.8 \\
Linolenic acid & 48 \\
Cholesterol (mg) & $<5$ \\
Protein (g) & 3 \\
Carbohydrate (g) & 19 \\
Dietary fiber (g) & 1 \\
Sugars (g) & 8 \\
Sodium (mg) & 60 \\
\hline
\end{tabular}

Amount per serving (serving size): 1 bar (30 g).

Total energy per serving is $130 \mathrm{kcal}$ of which $45 \mathrm{kcal}$ is obtained from fat.

\section{Preparation of Eri silkworm pupae snack bars}

Eri silkworm pupae snack bars were provided by Kasetsart Agricultural and Agro-Industrial Product Improvement Institute, Kasetsart University. The macronutrient profile of the Eri silkworm pupae snack bar was identical in composition and designed to contain $19 \mathrm{~g}$ of carbohydrate, $3 \mathrm{~g}$ of protein, and $5 \mathrm{~g}$ of fat. Each bar consisted of linolenic acid (approximately $4.17 \mathrm{~g} / 100 \mathrm{~g}$ ), leucine $(3.77 \mathrm{mg} / 100 \mathrm{~g})$, isoleucine $(2.22 \mathrm{mg} / 100 \mathrm{~g})$, and valine $(2.90 \mathrm{mg} / 100 \mathrm{~g})$, with a total sugar content of $26.67 \mathrm{~g} / 100 \mathrm{~g}$. The nutrition facts and the total fatty acid percentage in Eri snack bar are presented in Table 2. The placebo was prepared with a total energy amount equal to that of the Eri silkworm pupae snack bar.

\section{Exercise intervention}

The concurrent training program was carried out over three sessions a week, for $60 \mathrm{~min}$ per session. The exercise sessions included a 10-min warm-up with stretching followed by $40 \mathrm{~min}$ of concurrent (aerobic and resistance) exercise, and 10 min of stretching and cool-down exercises. The training program was designed according to the studies reviewed (Bartlett et al., 2011; Gibala et al., 2012; Kang and Ratamess, 2014). The training program was assessed the content validity by three experienced strength-training professionals. The content validity of the training program was good with index of item-objective congruence at 0.80 . Each training session was supervised by experienced trainers. The exer- cise intensity was monitored using a HR monitoring (H10, Polar electro Inc., Kempele, Finland) to ensure exercise consistency and safety. In the warm-up and the cool-down phases, participants performed stretching of the major muscle groups for $10 \mathrm{~min}$. Each stretch was sustained for between 15 and 30 sec to the point of tightness, and repeated 3 times.

Amid each session, the aerobic exercise was executed first, followed by a 30-sec rest interval prior to starting the resistance exercises (REs). The aerobic exercise consisted of 4-min low impact movement patterns including side-taps with punches to the side, leg curls with the elbows pulled back, knee-ups with raised arms, and rocking side to side with the arms raised. Aerobic intensity was maintained at between $70 \%$ and $80 \%$ of heart rate reserve.

REs were performed using an elastic band and subjects' BW. Participants were instructed to move through the full range of motion for each exercise. In session RE1, subjects performed two REs (bicep curls and lateral pull-downs) 2 sets of 8-12 repetitions. Sets and exercises were separated by 30 -sec rest intervals of active recovery. The exercises were completed at volitional speeds with subjects instructed to expire in the concentric phase, and inspire in the eccentric phase of the exercises. Detailed instructions were provided prior to the exercise sessions, although subjects were all experienced in resistance training. In sessions RE2, RE3, and RE4, the same aerobic exercise protocol was applied, though the REs were performed over two sets of 8-12 repetitions amid two exercises for each session according to the following order: shoulder press and squats, triceps extensions and lunges, push-ups, and planking, respectively.

\section{Anthropometrics and body composition measurements}

BW was measured via an electronic scale (Filizzola PL 150, Filizzola Ltda, São Paulo, Brazil). Height was measured minus shoes using a standard stadiometer (Health o Meter Professional, Sunbeam Products Inc., Boca Raton, FL, USA). BMI was calculated as weight divided by height squared. Waist circumference was measured from the midway between the lower rib margin and the iliac crest at the end of inspiration utilizing a flexible and inextensible measuring tape (Hoechstmass Balzer GmbH, Sulzbach, Hessen, Germany). Hip circumference was also measured at the level of trochanter major, with waist-to-hip circumference $(\mathrm{W} / \mathrm{H})$ ratio also calculated. Body composition and fat distribution were measured employing a DEXA scanner (Lunar iDXA, V17 software, GE Healthcare, Little Chalfont, Buckinghamshire, UK). Outcome variables were $\% \mathrm{BF}$, fat-free mass, $\mathrm{FM}$, and fat distribution. All measurements were conducted in a climate-controlled tempera- 
ture room according to the standard operating procedures recommended by the manufacturer.

\section{Blood samples and analysis}

Venous blood samples for biochemical analyses were taken in standardized fasting conditions in the mornings between 7:00 and 9:00 a.m. before and after intervention. Serum samples were stored frozen at $-80^{\circ} \mathrm{C}$ until analyzed. Serum concentrations of glucose, total cholesterol (TC), high-density lipoprotein cholesterol (HDL-C), and triglyceride (TG) were analyzed via the automated analyzer (Thermo Fischer Scientific Inc., Waltham, MA, USA). Low-density lipoprotein cholesterol (LDL-C) was calculated using the Friedewald equation (Friedewald et al., 1972). Plasma concentrations of high-sensitive $\mathrm{C}$-reactive protein (hs-CRP) were determined by an immunophelometric assay (BioTécnica Indústria e Comércio Ltda., Minas Gerais, Brazil). Serum fasting insulin concentrations were analyzed via the immunoassay analyzer (Siemens Healthcare diagnostics, Mannheim, Germany). The homeostasis model assessment of insulin resistance (HOMA-IR) index was calculated as (fasting insulin concentration $\times$ fasting glucose concentration)/ 22.5 (Matthews et al., 1985). Serum leptin was measured utilizing ELISA (DuoSet, R\&D Systems, Minneapolis, MN, USA). These methods are routinely employed at the Department of Pathology, Faculty of Medicine Ramathibodi Hospital, Mahidol University. All measurements were performed following 12-hr fasting at baseline and 7 days after the last training session to minimize any acute effects of exercise.

\section{Indirect calorimetry measurements}

Resting energy expenditure was measured via indirect calorimetry using a canopy method (Vmax Encore metabolic cart, Vyaire Medical Inc., Yorba Linda, CA, USA), calibrated according to the manufacturer's instructions prior to each use. Succeeding an overnight fast, participants were measured under standardized conditions in the morning between 6.00 and 10.00 a.m. (da Rocha et al., 2006). Upon arriving at the laboratory, participants rested for 20 min prior to the test, and adopted a relaxed position between testing. Expired air was collected to measure rates $(\mathrm{L} / \mathrm{min})$ of oxygen consumption $\left(\mathrm{VO}_{2}\right)$ and carbon dioxide production $\left(\mathrm{VCO}_{2}\right)$. Five min of steady-state data were included in the analysis and calculated for respiratory quotient and resting energy expenditure. Steady state is defined as a time period in which the mean $\mathrm{VO}_{2}$ and $\mathrm{VCO}_{2}$ vary by less than $10 \%$. Absolute resting energy expenditure ( $\mathrm{kcal} /$ day) was then calculated by applying the Weir equation (Weir, 1949).
Energy expenditure was also measured during submaximal exercise using metabolic cart. At least 1 week after the peak oxygen consumption $\left(\mathrm{VO}_{2 \text { peak }}\right)$ assessment, participants completed submaximal exercise on a treadmill (T-2100 Treadmill, GE Medical Systems, Wauwatosa, WI, USA); the first 3 min were performed at a speed of $2 \mathrm{mph}$ at $0 \%$ gradient, and the consecutive $10 \mathrm{~min}$ were performed at a relative speed equivalent to $40 \%$ and $60 \%$ of $\mathrm{VO}_{2 \text { peak. }}$. Expired air was collected from 3 to $5 \mathrm{~min}$, and 7 to $10 \mathrm{~min}$ to confirm steady-state. The $\mathrm{VO}_{2}$ and $\mathrm{VCO}_{2}(\mathrm{~L} / \mathrm{min})$ were used to calculate FAO $(\mathrm{g} / \mathrm{min})$ via the Peronnet and Massicotte equation (Peronnet and Massicotte, 1991): $\mathrm{FAO}=1.695 \mathrm{VO}_{2}-1.701 \mathrm{VCO}_{2}$. Accordingly, FAO was expressed as $\mathrm{g} /$ day, $\mathrm{kcal} / \mathrm{day}$, and percentage of energy expenditure derived from fat (\%) at rest, and $\mathrm{g} / \mathrm{min}$ during submaximal exercise. Energy expenditure was expressed as kcal/day at rest, $\mathrm{kcal} / \mathrm{min}$ during submaximal exercise, and per kilogram of BW, and fat-free mass. In addition, all participants replicated the percentage of macronutrient content of a 3-day food record completed prior to the baseline submaximal exercise test to control for effects of macronutrient content on substrate oxidation.

\section{HRV recordings and analysis}

The R-R intervals were recorded using a 5-min electrocardiogram (ECG) recording (eMotion Faros device, Mega Electronics, Kuopio, Finland) in the lying position. The ECG recording was collected online at a sampling rate of $1,000 \mathrm{~Hz}$, in real time and stored on a computer. The ECG recording took place at a fixed time in the morning (between 8:00 and 10:00 a.m.) to avoid possible circadian influences on autonomic function. Prior to the recording, participants rested comfortably in the supine position for at least $20 \mathrm{~min}$ in a quiet, thermoneutral $\left(23^{\circ} \mathrm{C}-25^{\circ} \mathrm{C}\right)$ room. Respiratory rate, determined by visual inspection of chest movement, was within the normal range (12-20/min).

The R-R intervals were analyzed via a software program using the recommendations of the Task Force of the European Society of Cardiology as well as the North American Society of Pacing and Electrophysiology (1996). Time- and frequency-domain parameters together with nonlinear components of HRV were calculated. The time-domain parameters consisted of mean R-R intervals, standard deviation of normal R-R intervals (SDNN), and squared differences between adjacent normal N-N intervals (RMSSD). The frequency-domain parameters consisted of very low-frequency power (VLF power 0.001-0.04 Hz), low-frequency power (LF power: $0.04-0.15 \mathrm{~Hz}$ ), and high-frequency power (HF power: $0.15-0.4 \mathrm{~Hz}$ ). For the frequency-domain analysis, VLF power spectrum could be an indicator of sympathetic thermoregulatory 
and metabolic energy signaling (Fujibayashi et al., 2009; Nagai et al., 2005). Nonlinear HRV components, Poincaré plot standard deviation perpendicular the line of identity (SD1) and along the line of identity (SD2), were analyzed quantitatively by calculating the standard deviations from the R-R interval data.

\section{Statistical analysis}

Results were expressed as mean and standard deviation. Normality was assessed by employing the Shapiro-Wilk test. Baseline comparisons between groups were performed using one-way analysis of variance. Baseline values were employed as covariates. If necessary, the data were transformed logarithmically prior to analysis of covariance (ANCOVA) in order to fulfill the criterion of normal distribution. Besides that, measurement outcomes were tested to determine whether they met the assumptions of normality, linearity, and homogeneity of variance. Outcome variable comparisons between groups were performed through ANCOVA measures. Pairwise comparisons were performed by applying the Bonferroni test. Within-group analyses were carried out with dependent t-test. The effect size (ES) was calculated between each pair of measurements according to Cohen (1988). Pearson's correlation was applied to assess the relationships between fluctuations in energy expenditure, FAO and HRV variables following adjustment for baseline values as a confounding factor. Statistical significance was set at $P<0.05$. Statistical analyses were conducted by employing IBM SPSS Statistics ver. 26.0 (IBM Co., Armonk, NY, USA).

\section{RESULTS}

Subjects' characteristics were not significantly different between groups. All groups had similar profiles amid all clinical parameters examined (see Table 1). Average energy intake during the experiment did not vary significantly among groups. Mean energy intakes of the ERI and the CBT-ERI were 1,871 \pm 360 and 1,884 \pm $228 \mathrm{kcal} /$ day at baseline, and $1,920 \pm 423$ and $1,877 \pm 483 \mathrm{kcal} /$ day at the conclusion of the interventions, respectively; the corresponding data of the CON was $1,811 \pm 270 \mathrm{kcal} /$ day and $1,826 \pm 294$ $\mathrm{kcal} /$ day. Carbohydrates provided approximately $51 \%$; fat approximately $30 \%$; and protein approximately $19 \%$ of participants' daily energy intake in all groups. These percentages did not alter prior to and post experimentation. The levels of habitual physical activity besides the interventions did not change significantly over time, and no difference was observed between groups. Exercise attendance of the CBT-ERI was $85.4 \% \pm 5 \%$. For exercise sessions, participants completed $99 \% \pm 2.6 \%$ of the prescribed exercise du- ration and exercised at $96.9 \% \pm 7.4 \%$ of the prescribed exercise intensity throughout the training period. There were no apparent side effects induced by the Eri silkworm pupae throughout the study.

Anthropometrics and body composition at baseline and following the interventions are demonstrated in Table 1. No significant differences were observed amid any anthropometrics and body composition after the intervention in the CON. In the ERI, waist circumference $(-3.2 \%, \mathrm{ES}=0.27, P<0.05)$ and hip circumference $(-2.2 \%, \mathrm{ES}=0.22, P<0.05)$ were significantly reduced following the intervention, with no significant differences among groups. What's more, there were no significant differences in terms of body composition and fat distribution compared to baseline following the intervention.

The CBT-ERI rendered reductions in $\mathrm{BW}(-2.4 \%, \mathrm{ES}=0.28$, $P<0.05)$, BMI $(-2.9 \%, \mathrm{ES}=0.26, P<0.01), \% \mathrm{BF}(-3.5 \%, \mathrm{ES}=$ $0.22, P<0.05), \mathrm{FM}(-6.1 \%, \mathrm{ES}=0.37, P<0.01)$, arm $(-4.7 \%$, ES $=$ $0.40, P<0.05)$, leg $(-2.5 \%, \mathrm{ES}=0.16, P<0.05)$, trunk $(-2.4 \%$, $\mathrm{ES}=0.34, P<0.05)$, gynoid $(-4.2 \%, \mathrm{ES}=0.30, P<0.05)$, and android $(-9.3 \%, \mathrm{ES}=0.22, P<0.05) \mathrm{FM}$, waist circumference $(-5.1$, $\mathrm{ES}=0.77, P<0.01)$, hip circumference $(-2.1 \%, \mathrm{ES}=0.54, P<0.05)$, and $\mathrm{W} / \mathrm{H}$ ratio $(-3 \%, \mathrm{ES}=0.20, P<0.05)$ following the intervention. Moreover, trunk and gynoid FM in the CBT-ERI were significantly lower than in the CON (ES $=0.15, P<0.05$ and $\mathrm{ES}=$ $0.09, P<0.05$ for trunk and gynoid FM, respectively) as well as the ERI (ES $=0.02, P<0.05$ and $\mathrm{ES}=0.16, P<0.05$ for trunk and gynoid FM, respectively) following intervention (Table 1).

Table 1 indicates the results for cardiometabolic risk markers at baseline and post interventions. No significant variances were observed in leptin, HDL-C concentrations, TC/HDL-C ratio, and LDL-C/HDL-C ratio following the interventions in all groups. Succeeding the interventions, the CON exhibited significant increments in fasting blood glucose $(11.8 \%, \mathrm{ES}=0.63, P<0.01)$, insulin $(26.4 \%, \mathrm{ES}=0.57, P<0.05)$, HOMA-IR $(41.3 \%, \mathrm{ES}=0.67$, $P<0.05)$, and LDL-C $(14.9 \%, \mathrm{ES}=0.47, P<0.05)$ concentrations, though with no significant dissimilarities among groups. The ERI exhibited significant increments in fasting blood glucose $(19.1 \%$, $\mathrm{ES}=0.36, P<0.01)$, insulin $(36.6 \%, \mathrm{ES}=0.69, P<0.05)$, HOMAIR (63.2\%, ES $=0.66, P<0.01)$, TC (9.7\%, $\mathrm{ES}=0.34, P<0.05)$, TG $(40.4 \%, \mathrm{ES}=0.55, P<0.05)$, and LDL-C $(14.9 \%, \mathrm{ES}=0.59$, $P<0.01)$ concentrations, and reduction in hs-CRP concentration $(-26.2 \%, \mathrm{ES}=0.46, P<0.05)$ following the interventions, yet with no significant differences among groups.

Just the CBT-ERI brought about reductions in hs-CRP concentration of $26.1 \%(\mathrm{ES}=0.75, P<0.05)$ and TC concentration of 
Table 3. Energy expenditure and fat oxidation at rest and during submaximal exercise at baseline and post interventions

\begin{tabular}{|c|c|c|c|c|c|c|}
\hline \multirow{2}{*}{ Variable } & \multicolumn{2}{|c|}{$\operatorname{CON}(n=12)$} & \multicolumn{2}{|c|}{$E R I(n=2)$} & \multicolumn{2}{|c|}{ CBT-ERI $(n=12)$} \\
\hline & Baseline & Posttest & Baseline & Posttest & Baseline & Posttest \\
\hline \multicolumn{7}{|l|}{ Resting } \\
\hline Energy expenditure (kcal/day) & $1,348.9 \pm 168.9$ & $1,351.8 \pm 220.7$ & $1,248.4 \pm 210.3$ & $1,278.3 \pm 218.7$ & $1,308.7 \pm 143.8$ & $1,404.5 \pm 187.7^{*}$ \\
\hline Energy expenditure (kcal/day/kg BW) & $19.3 \pm 2.7$ & $19.3 \pm 2.6$ & $18.6 \pm 1.9$ & $19 \pm 1.9$ & $17.9 \pm 1.8$ & $19.5 \pm 2.3^{*, \dagger}$ \\
\hline Energy expenditure (kcal/day/kgFFM) & $32.2 \pm 3.5$ & $32 \pm 4.5$ & $31.4 \pm 2.9$ & $31.9 \pm 2.2$ & $29.5 \pm 4$ & $31.6 \pm 3.9^{*}$ \\
\hline RER & $0.79 \pm 0.07$ & $0.79 \pm 0.05$ & $0.78 \pm 0.04$ & $0.77 \pm 0.03$ & $0.77 \pm 0.06$ & $0.76 \pm 0.03$ \\
\hline $\mathrm{FAO}(\mathrm{g} / \mathrm{min})$ & $0.07 \pm 0.03$ & $0.07 \pm 0.02$ & $0.07 \pm 0.02$ & $0.07 \pm 0.2$ & $0.07 \pm 0.06$ & $0.08 \pm 0.02$ \\
\hline FAO (kcal/day) & $883 \pm 331.9$ & $906.1 \pm 263.9$ & $871.1 \pm 291.9$ & $930.7 \pm 218.6^{*}$ & $929.5 \pm 330.6$ & $1,056.9 \pm 224.7^{*,+}$ \\
\hline FAO $(\%)$ & $65.7 \pm 22.9$ & $67.9 \pm 16.8$ & $68.7 \pm 14$ & $72.9 \pm 11.6$ & $70.6 \pm 22$ & $75.1 \pm 10.9$ \\
\hline \multicolumn{7}{|l|}{$40 \%$ of $\mathrm{VO}_{2 \text { peak }}$} \\
\hline Energy expenditure (kcal/min) & $0.5 \pm 0.2$ & $0.6 \pm 0.2$ & $0.4 \pm 0.1$ & $0.4 \pm 0.2$ & $0.7 \pm 0.3$ & $0.7 \pm 0.3$ \\
\hline RER & $0.79 \pm 0.1$ & $0.81 \pm 0.1$ & $0.78 \pm 0.05$ & $0.76 \pm 0.05$ & $0.86 \pm 0.07$ & $0.83 \pm 0.05$ \\
\hline FAO (g/min) & $0.19 \pm 0.1$ & $0.17 \pm 0.1$ & $0.21 \pm 0.1$ & $0.21 \pm 0.1$ & $0.18 \pm 0.1$ & $0.24 \pm 0.1^{*, t}$ \\
\hline \multicolumn{7}{|l|}{$60 \%$ of $\mathrm{VO}_{2 \text { peak }}$} \\
\hline Energy expenditure (kcal/min) & $0.9 \pm 0.3$ & $1 \pm 0.5$ & $0.7 \pm 0.2$ & $0.8 \pm 0.3$ & $1.3 \pm 0.5$ & $1.2 \pm 0.5$ \\
\hline RER & $0.88 \pm 0.1$ & $0.9 \pm 0.1$ & $0.85 \pm 0.1$ & $0.85 \pm 0.1$ & $0.86 \pm 0.1$ & $0.84 \pm 0.0^{*}$ \\
\hline $\mathrm{FAO}(\mathrm{g} / \mathrm{min})$ & $0.23 \pm 0.1$ & $0.19 \pm 0.1$ & $0.23 \pm 0.1$ & $0.23 \pm 0.1$ & $0.18 \pm 0.1$ & $0.24 \pm 0.1^{* *}$ \\
\hline
\end{tabular}

Values are presented as mean \pm standard deviation.

CON, control group; ERI, Eri silkworm pupae ingestion; CBT-ERI, combined concurrent training and Eri silkworm pupae ingestion; BW, body weight; FFM, fat-free mass; RER, respiratory exchange ratio; $\mathrm{FAO}$, fat oxidation rate; $\mathrm{VO}_{2 \text { peak, }}$, peak oxygen consumption.

${ }^{*} P<0.05$ and ${ }^{* *} P<0.01$ to within-group comparison (baseline vs. post interventions). ${ }^{\dagger} P<0.05$ to between-group comparison (vs. CON).

$10.6 \%$ (ES $=0.72, P<0.01$ ) following the interventions. Moreover, hs-CRP concentration $(\mathrm{ES}=0.25, P<0.05)$ in the CBT-ERI was significantly lower than the ERI following the experimentations. TC concentration in the CBT-ERI was significantly lower than the $\mathrm{CON}(\mathrm{ES}=0.66, P<0.01)$, as well as the $\mathrm{ERI}(\mathrm{ES}=0.43$, $P<0.01)$ following the experimentations. What's more, there were no significant differences in fasting blood glucose, insulin, HOMA-IR, TG, LDL-C, and HDL-C concentrations compared to baseline following the intervention (Table 1).

Table 3 indicates the results for energy expenditure and FAO at rest and during submaximal exercise following the interventions. No significant variances were detected in energy expenditure and FAO at rest and during submaximal exercise following the intervention in the CON. In the ERI, resting FAO was significantly increased $(11.5 \%, \mathrm{ES}=0.23, P<0.05)$ succeeding the intervention, though with no significant differences among groups. There were no significant differences in resting energy expenditure, energy expenditure, and $\mathrm{FAO}$ during submaximal exercise compared to baseline following the experimentation.

Just the CBT-ERI brought about increments in resting energy expenditure $(\mathrm{kcal} / \mathrm{day} / \mathrm{kg} \mathrm{BW})(7.8 \%, \mathrm{ES}=0.78, P<0.05)$, resting $\mathrm{FAO}(24.5 \%, \mathrm{ES}=0.45, P<0.05)$, FAO during submaximal exercise at an intensity of $40 \%$ of $\mathrm{VO}_{2 \text { peak }}(72.7 \%, \mathrm{ES}=0.60, P<0.05)$ and $60 \%$ of $\mathrm{VO}_{2 \text { peak }}(60.4 \%, \mathrm{ES}=0.60, P<0.01)$, and reduction in RER $(-3.5 \%, \mathrm{ES}=0.60, P<0.05)$ during submaximal exercise at an intensity of $60 \%$ of $\mathrm{VO}_{2 \text { peak }}$ following the intervention. Moreover, resting energy expenditure in the CBT-ERI was significantly higher than the $\mathrm{CON}(\mathrm{ES}=0.08, P<0.05)$ post experimentations. $\mathrm{FAO}$ at rest and during submaximal exercise at an intensity of $40 \%$ of $\mathrm{VO}_{2 \text { peak }}$ in the CBT-ERI were significantly higher than the $\mathrm{CON}$ (ES $=0.62, P<0.05$ and $\mathrm{ES}=0.70, P<0.05$, respectively) post experimentations (Fig. 1). However, there were no significant differences in energy expenditure during submaximal exercise compared to baseline following the intervention (Table 3).

HRV variables at baseline and succeeding the interventions are demonstrated in Table 4. No significant differences were observed in any HRV variables after the intervention in the CON; except for VLF power. VLF power $(-6.9 \%, \mathrm{ES}=0.77, P<0.05)$ was significantly lower in the CON compared to baseline, with no noteworthy differences among groups. Succeeding the interventions, the ERI exhibited a significant increment in InVLF power (14.6\%, $\mathrm{ES}=1.38, P<0.05)$ compared to baseline. Moreover, the increment in $\operatorname{lnVLF}$ power $(\mathrm{ES}=1.17, P<0.01)$ was significantly greater compared to the $\mathrm{CON}$ following the interventions. However, there were no significant differences in term of time-domain and nonlinear variables of $\mathrm{HRV}$ compared to baseline following the intervention. 

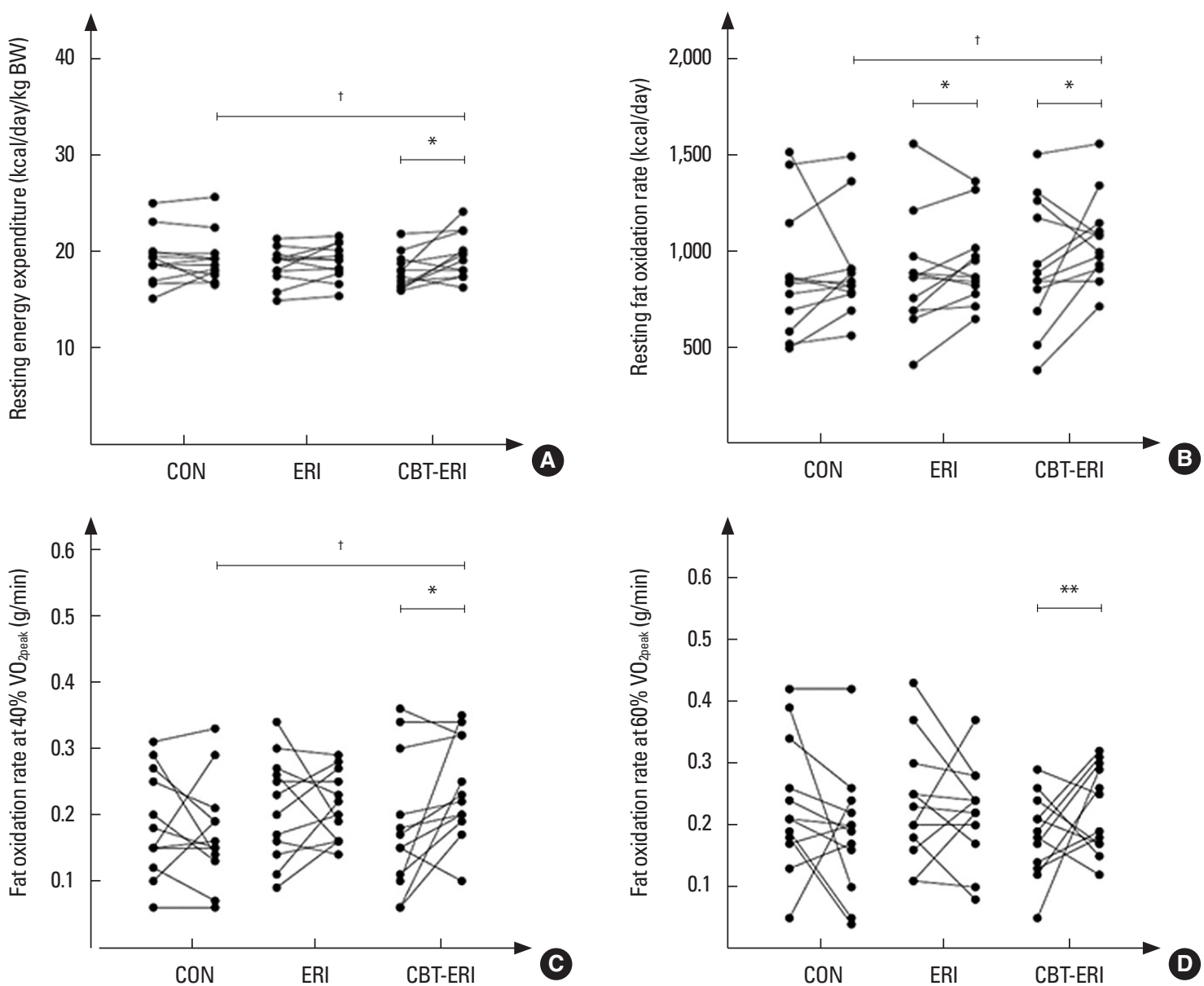

Fig. 1. Individual data of resting energy expenditure (A), resting fat oxidation rate (B), fat oxidation rate during submaximal exercise at an intensity of $40 \%$ of $\mathrm{VO}_{2 \text { peak }}$ (C), fat oxidation rate during submaximal exercise at an intensity of $60 \%$ of $\mathrm{VO}_{2 \text { peak }}$ (D) at baseline and following the interventions. CON, control group; ERI, Eri silkworm pupae ingestion; $\mathrm{CBT}-\mathrm{ERI}$, combined concurrent training and Eri silkworm pupae ingestion; $\mathrm{VO}_{2 \text { peak, }}$ peak oxygen consumption. ${ }^{*} P<0.05$ and ${ }^{* *} P<0.01$ to within-group comparison (baseline vs. post interventions). ${ }^{\dagger} P<0.05$ to between-group comparison (vs. CON).

Only the CBT-ERI brought about increments in SDNN $(58.3 \%$, $\mathrm{ES}=0.97, P<0.01)$, pNN50 $(423.8 \%, \mathrm{ES}=1.06, P<0.01)$, and $\operatorname{lnVLF}$ power $(8.3 \%, \mathrm{ES}=0.63, P<0.05)$ following experimentation. Furthermore, $\mathrm{SDNN}(\mathrm{ES}=1.14, P<0.01)$, $\mathrm{pNN} 50$ ( $\mathrm{ES}=0.87$, $P<0.05$ ), and $\operatorname{lnVLF}$ power ( $\mathrm{ES}=0.88, P<0.01$ ) in the CBT-ERI were significantly greater than the $\mathrm{CON}$ following the interventions. SDNN (ES=0.79, $P<0.05)$ in the CBT-ERI was also significantly greater than the ERI following the interventions. However, there were no significant differences in term of nonlinear variables of HRV compared to baseline following the intervention (Table 4).

The increment of relative resting energy expenditure (kcal/day/ $\mathrm{kg} \mathrm{BW}$ ) in the CBT-ERI was positively related to the change in rate of FAO (kcal/day) at rest $(r=0.643, P=0.001)$ and during submaximal exercise $(\mathrm{g} / \mathrm{min})$ at an intensity of $40 \%$ of $\mathrm{VO}_{2 \text { peak }}$ $(r=0.435, P=0.034)$, and $\operatorname{lnVLF}$ power $(r=0.421, P=0.045)$ (Fig. $2 \mathrm{~A})$; meanwhile, the increment of relative resting energy expenditure was negatively related to the alteration in BMI $(r=-0.526$, $P=0.008)$ and TC concentration $(r=-0.411, P=0.046)$ following the intervention. The increment of resting FAO in the CBT-ERI was positively correlated with the alteration in SDNN $(r=0.409$, $P=0.047)$ and $\operatorname{lnVLF}$ power $(r=0.448, P=0.028)$ (Fig. $2 \mathrm{~B})$ succeeding the intervention. Nevertheless, the association with the increment of resting FAO in the ERI did not reach statistical significance following the intervention.

\section{DISCUSSION}

The hypothesis of this study was that the combination of concurrent training with Eri silkworm pupae intake could improve 
Table 4. Heart rate variability at baseline and post interventions

\begin{tabular}{|c|c|c|c|c|c|c|}
\hline \multirow{2}{*}{ Variable } & \multicolumn{2}{|c|}{$\operatorname{CON}(n=12)$} & \multicolumn{2}{|c|}{ ERI $(n=12)$} & \multicolumn{2}{|c|}{ CBT-ERI $(n=12)$} \\
\hline & Baseline & Posttest & Baseline & Posttest & Baseline & Posttest \\
\hline RR intervals (ms) & $819.6 \pm 121$ & $766.6 \pm 95.7$ & $810.9 \pm 122.5$ & $810.2 \pm 88$ & $854.7 \pm 87.7$ & $897.9 \pm 113.2$ \\
\hline SDNN (ms) & $41.5 \pm 19.4$ & $39.2 \pm 19.8$ & $45 \pm 18.9$ & $49.8 \pm 15.9$ & $45.7 \pm 14.4$ & $70.5 \pm 33.3^{* *, t t, \pm}$ \\
\hline pNN50 (\%) & $11.5 \pm 16$ & $7 \pm 14.6$ & $12.1 \pm 16$ & $11.7 \pm 11.7$ & $7.2 \pm 6.7$ & $20.6 \pm 16.6^{* *, t}$ \\
\hline RMSSD (ms) & $31 \pm 21.6$ & $37.2 \pm 26.9$ & $37.5 \pm 24$ & $37.3 \pm 14.7$ & $61.3 \pm 70.6$ & $60 \pm 33.8$ \\
\hline Total power $\left(\mathrm{ms}^{2}\right)$ & $1,988.4 \pm 1,824.3$ & $1,911.9 \pm 1,781.7$ & $2,194.4 \pm 2,353.7$ & $2,894.4 \pm 1,739.3$ & $2,327.9 \pm 2,529.2$ & $3,082.5 \pm 2,885.2$ \\
\hline $\mathrm{HF}\left(\mathrm{ms}^{2}\right)$ & $580.4 \pm 809.8$ & $754.6 \pm 917.1$ & $895.7 \pm 1,179.2$ & $825 \pm 832.4$ & $1,137.2 \pm 2,541.6$ & $1,049.3 \pm 1,063.3$ \\
\hline $\operatorname{lnHF}\left(\mathrm{ms}^{2}\right)$ & $5.6 \pm 1.4$ & $5.7 \pm 1.7$ & $5.9 \pm 1.5$ & $6.1 \pm 1.2$ & $6.1 \pm 1.1$ & $6.5 \pm 1.2$ \\
\hline$H F(n u)$ & $27.3 \pm 17.5$ & $33.6 \pm 18.7$ & $31.4 \pm 17.5$ & $24.1 \pm 15.1$ & $32.3 \pm 21.8$ & $31.5 \pm 17.7$ \\
\hline$L F\left(\mathrm{~ms}^{2}\right)$ & $497.8 \pm 645$ & $630.9 \pm 583.1$ & $675.6 \pm 922.1$ & $639.4 \pm 466.6$ & $452.2 \pm 278.3$ & $788.8 \pm 783$ \\
\hline $\ln L F\left(m s^{2}\right)$ & $5.5 \pm 1.3$ & $5.7 \pm 1.7$ & $6 \pm 1$ & $6.1 \pm 1$ & $5.9 \pm 0.8$ & $6.3 \pm 1$ \\
\hline$L F(n u)$ & $21.2 \pm 9.7$ & $31.1 \pm 11$ & $28.3 \pm 9.3$ & $21.4 \pm 10.7$ & $24.6 \pm 13.1$ & $23.7 \pm 9.6$ \\
\hline $\operatorname{VLF}\left(\mathrm{ms}^{2}\right)$ & $910.2 \pm 837.1$ & $526.5 \pm 461.1$ & $623.1 \pm 469.6$ & $1,429.9 \pm 1,072.4$ & $738.5 \pm 573.5$ & $1,244.4 \pm 1,362.3$ \\
\hline $\operatorname{lnVLF}\left(\mathrm{ms}^{2}\right)$ & $6.3 \pm 1.1$ & $5.8 \pm 1.4$ & $6.2 \pm 0.6$ & $7.1 \pm 0.7^{*,+t}$ & $6.3 \pm 0.8$ & $6.8 \pm 0.8^{*, t \dagger}$ \\
\hline $\operatorname{VLF}(n u)$ & $51.5 \pm 22.9$ & $35.3 \pm 18.8^{*}$ & $40.2 \pm 19.5$ & $54.5 \pm 20.5$ & $43.1 \pm 22.3$ & $44.6 \pm 17.8$ \\
\hline LF/HF & $1.2 \pm 1.1$ & $1.8 \pm 2.3$ & $1.4 \pm 1.5$ & $1.3 \pm 1.4$ & $1.2 \pm 0.7$ & $1.1 \pm 0.9$ \\
\hline SD1 & $21.9 \pm 15.3$ & $26.3 \pm 19$ & $26.5 \pm 16.9$ & $26.4 \pm 10.4$ & $43.3 \pm 49.9$ & $42.4 \pm 23.9$ \\
\hline SD2 & $54 \pm 24.1$ & $48 \pm 22.4$ & $57.2 \pm 22.5$ & $64.9 \pm 21.1$ & $63.6 \pm 22.4$ & $70.4 \pm 24$ \\
\hline SD2/SD1 & $2.9 \pm 1.2$ & $2.2 \pm 0.9$ & $2.7 \pm 1.3$ & $2.6 \pm 0.9$ & $2.2 \pm 1.2$ & $2.1 \pm 1$ \\
\hline
\end{tabular}

Values are presented as mean \pm standard deviation.

CON, control group; ERI, Eri silkworm pupae ingestion; CBT-ERI, combined concurrent training and Eri silkworm pupae ingestion; SDNN, standard deviation of normal R-R intervals; pNN50, percentage difference between adjacent normal R-R intervals $>50 \mathrm{~ms}$; RMSSD, square root of the mean squared difference between adjacent normal R-R intervals; HF, high-frequency power; LF, low-frequency power; VLF, very low-frequency power; LF/HF, low-frequency to high-frequency ratio; In, natural logarithm; SD, Poincaré plot standard deviation perpendicular the line of identity; SD2, Poincaré plot standard deviation along the line of identity; SD2/SD1, SD2 to SD1 ratio.

${ }^{*} P<0.05$ and ${ }^{* *} P<0.01$ to within-group comparison (baseline vs. post interventions). ${ }^{\dagger} P<0.05$ and ${ }^{\dagger t} P<0.01$ to between-group comparison (vs. CON). ${ }^{\ddagger} P<0.05$ to between-group comparison (vs. ERI).
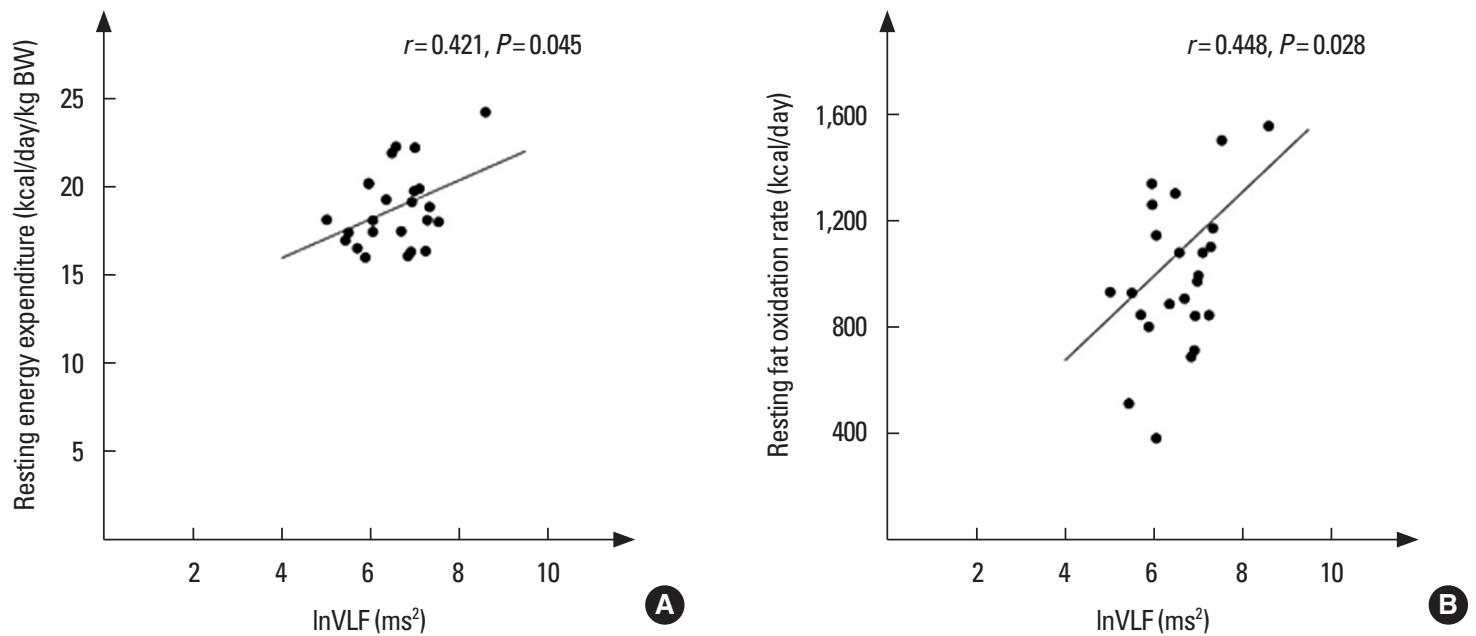

Fig. 2. The correlation between fluctuations in resting energy expenditure (A) and InVLF power, resting fat oxidation rate (B) and InVLF power, in the CBT-ERI. InVLF, the natural logarithm of very low-frequency power band of heart rate variability; BW, body weight; CBT-ERl, combined concurrent training and Eri silkworm pupae ingestion.

resting energy expenditure, FAO at rest and during submaximal exercise, as well as cardiometabolic risk markers in overweight and obese adults. The primary findings were that Eri silkworm pupae ingestion alone induced significant modifications in resting 
FAO and lnVLF power band of HRV. Adding three weekly sessions of concurrent training to this approach delivers better outcomes than Eri silkworm pupae ingestion alone; not only the modifications in resting FAO and lnVLF power band of HRV, but also resting energy expenditure and FAO during submaximal exercise were also increased.

Moreover, the results demonstrated the significant relationship among resting energy expenditure, FAO, and lnVLF power band of HRV following 8 weeks of the combined intervention. What's more, our results demonstrated a favorable impact of combined concurrent training and Eri silkworm pupae intake on trunk and gynoid FM, corresponding to an adaptation of both resting energy expenditure and $\mathrm{FAO}$ at rest and during submaximal exercise. For overweight and obese individuals an increased energy expenditure and FAO may be beneficial in order to reduce BW.

Very little is known about the role of combined concurrent training and Eri silkworm pupae intake with respect to energy expenditure and FAO at rest and during exercise. The majority of studies conducted to date have focused on concurrent training alone (Schwingshackl et al., 2013) or PUFAs supplementation alone (Fujibayashi et al., 2009; Kien et al., 2005; Nagai et al., 2005; Piers et al., 2002). Improvements in energy expenditure and/or FAO after a high ratio of PUFAs to saturated fatty acids ingestion have previously been reported (Kien et al., 2005; Piers et al., 2002). Eri silkworm pupae are enriched with PUFAs accounting for approximately $70 \%$ of the total fatty acids (Longvah et al., 2012). Our findings are consistent with previous studies (Fujibayashi et al., 2009; Nagai et al., 2005) whereby they reported the same effects of PUFAs ingestion on resting energy expenditure and FAO. The previous studies suggested that PUFAs ingestion might affect resting energy expenditure and FAO via activated sympathetic thermogenesis. An augmentation of sympathetic thermogenesis seems to be a part of an energy regulation system which burns excessive energy in the mode of heat production.

Moreover, the VLF power band of HRV has been introduced as an indicator of sympathetic thermoregulatory and metabolic energy signaling (Millis et al., 2011). Our results also indicated the significant increase in resting FAO and lnVLF power following Eri silkworm pupae intake. Nevertheless, according to our revelations, the correlation between resting FAO and lnVLF power in the ERI did not reach statistical significance. Thus, the increase in resting FAO post Eri silkworm pupae intake may be achieved via other mechanisms such as faster gastric emptying (Robertson et al., 2002), increased intestinal absorption, and preferential hepatic oxidation (DeLany et al., 2000). Peculiarly, PUFAs have been shown to be effective in up-regulating PPAR a expression, and stimulating the transcription of genes involved in $\mathrm{FAO}$ and thermogenesis while suppressing the genes regulating fatty acid synthesis (Kliewer et al., 1997).

What's more, our findings indicate that the combined intervention delivers better outcomes than Eri silkworm pupae ingestion alone. Hence, a noteworthy statistical correlation among resting energy expenditure, FAO, and InVLF power in the CBT-ERI succeeding the combined intervention was observed. A possible mechanism responsible for increased resting energy expenditure and FAO at rest and during exercise in the CBT-ERI may be an improved sympathetic thermogenesis as the important statistical correlation among resting energy expenditure, FAO, and $\operatorname{lnVLF}$ has been detected.

Another possible mechanism may be related to improved parasympathetic activity. The present study demonstrated that the CBT-ERI brought about increases in vagal-related HRV parameters (SDNN and pNN50). A noteworthy statistical correlation between increased resting FAO and SDNN was also observed. Vagal activity plays an important role in the regulation of caloric-intake behavior and metabolic homeostasis. Moreover, an increase in vagal activity relates to the thermic effect of food. In obese individuals, the reductions of both sympathetic and vagal activity have been reported to be linked with low resting energy expenditure and high body fat (Messina et al., 2017). The modulation of both sympathetic and vagal activities provides a complex homeostatic mechanism enabling the regulation of energy balance (Villarroya and Vidal-Puig, 2013).

In addition, our results showed that FAO during submaximal exercise increased in only the CBT-ERI with no change in the ERI. The combined concurrent training and Eri silkworm ingestion have positive effects on lowering RER value during submaximal exercise $\left(60 \%\right.$ of $\left.\mathrm{VO}_{2 \text { paak }}\right)$ indicating an increase in $\mathrm{FAO}$. Increased FAO during exercise can be associated with the ability of skeletal muscles to mobilize and oxidize free fatty acids as well as the activity of pyruvate dehydrogenase and glycogenolysis (Yeo et al., 2011).

Taking all the above results into account, it is thought that PUFAs contained in large quantities in the Eri silkworm pupae, especially linolenic acid, can inhibit fat storage by accelerating fat catabolism. Moreover, the combination of concurrent training and Eri silkworm pupae ingestion may conceivably suppress obesity via an increase in resting energy expenditure through a significant increase in FAO at rest and during exercise. This suggests that concurrent training together with Eri silkworm pupae intake could 
generate synergistic effects enhancing resting energy expenditure and $\mathrm{FAO}$ at rest and during submaximal exercise among obese adults. Improvements to resting energy expenditure and FAO are essential due to their significant roles in reducing cardiometabolic risk factors (Rosenkilde et al., 2010).

As for body composition, we also discovered that a combined intervention is more effective than Eri silkworm pupae ingestion alone, in terms of reducing BW, BMI, \%BF, FM, especially trunk and gynoid FM, waist circumference, hip circumference, and $\mathrm{W} / \mathrm{H}$ ratio. Besides that, we discovered a significant correlation between resting energy expenditure and BMI in the CBT-ERI. The favorable impact of the combined interventions on BMI and body fat corresponds to an adaptation of both resting energy expenditure and FAO at rest and during submaximal exercise. On the other hand, the ERI did not change in body composition. This is consistent with the lack of increased resting energy expenditure. These findings also indicate that trunk and gynoid FM were effectively modified by the combined intervention. More marked reductions of these fat areas in the CBT-ERI showed a greater effect of the combined intervention on subcutaneous adipose tissue. Such remarkable observed reductions can be explained as an outcome of increased resting energy expenditure.

As for lipid profiles, noting that our participants did not change their caloric intake through the study, only a reduction in TC concentration was observed in the CBT-ERI. Our results demonstrate that the improvements in $\mathrm{TC}$ are directly related to the increase in resting energy expenditure. Hence the results clearly show that combined concurrent training and Eri silkworm pupae ingestion attenuate hypercholesterolemia more effectively than intake of Eri silkworm pupae alone. Attenuation of hypercholesterolemia by the combined intervention may be attributed to the improvement of cardiometabolic risk factors in obese adults.

Regarding inflammatory markers, hs-CRP, which is a surrogate marker of global vascular health, decreased in both the ERI and the CBT-ERI. Reduction in hs-CRP level has been reported to be associated with exercise training as well as dietary PUFAs (Ortega et al., 2016; Tartibian et al., 2011). A reduction in hs-CRP level in metabolic syndrome patients was reported following a combination of exercise training and PUFAs ingestion (Ortega et al., 2016). Similarly, a previous study reported that the levels of proinflammatory cytokines in postmenopausal women were decreased after a combined exercise training and PUFAs supplementation (Tartibian et al., 2011). Furthermore, we discovered the significant correlation between reduction in hs-CRP levels and BW, BMI, waist circumference, and $\mathrm{W} / \mathrm{H}$ ratio following the combined in- tervention. Consistent with our findings, a previous study has suggested that the change in hs-CRP correlated similarly with changes in BW and all measures of body fat, including total body fat, and total abdominal, subcutaneous abdominal, and visceral fat (Fisher et al., 2011).

There are some study limitations to be considered. In this particular study design, there was no concurrent training group, thus signifying a lack of trained control groups. Furthermore, the assessment of sympathetic and parasympathetic activities through spectral analysis of HRV only, should be interpreted with caution. Although, the association between the fluctuations in resting energy expenditure, FAO, and lnVLF power were detected, the correlation coefficient is weak. Further investigations on the possible energy expenditure-promoting effects of the combined intervention are recommended. Finally, our study incorporated a low number of subjects which stands as a significant limitation. Thus, it is possible that a larger sample size could result in more precisely yielded effects and outcomes amid intervention. Hence, in terms of future researches, such studies ought to be addressed with a larger study population.

In conclusion, this study demonstrates that concurrent training together with dietary Eri silkworm pupae for 8 weeks leads to an increased resting energy expenditure and $\mathrm{FAO}$ at rest and during submaximal exercise, as well as reduced trunk and gynoid FM. Although Eri silkworm pupae ingestion did not directly affect body composition, it may augment the effects of concurrent training on body fat by increasing resting FAO. The combined concurrent training and Eri silkworm pupae intake have additional favorable effects on blood cholesterol and inflammatory marker. These results suggest that the combined interventions of concurrent training and dietary Eri silkworm pupae may prevent or lessen obesity and possibly reduce the risk of cardiometabolic disease.

\section{CONFLICT OF INTEREST}

No potential conflict of interest relevant to this article was reported.

\section{ACKNOWLEDGMENTS}

This research is supported in part by the Graduate Program Scholarship from the Graduate School, Kasetsart University in 2018. The study is also funded by Thailand Science Research and Innovation in 2019 (grant No. RDG62T0041). We wish to thank the participants for their enthusiastic participation throughout 
the study. We would also like to sincerely thank the Department of Pathology, Faculty of Medicine Ramathibodi Hospital, Mahidol University for determining blood chemistry measurements. Finally, we wish to show our appreciation to Kasetsart Agricultural and Agro-Industrial Product Improvement Institute for products, preparation, and analysis.

\section{REFERENCES}

Albracht-Schulte K, Kalupahana NS, Ramalingam L, Wang S, Rahman SM, Robert-McComb J, Moustaid-Moussa N. Omega-3 fatty acids in obesity and metabolic syndrome: a mechanistic update. J Nutr Biochem 2018;58:1-16.

Bartlett JD, Close GL, MacLaren DP, Gregson W, Drust B, Morton JP. Highintensity interval running is perceived to be more enjoyable than moderate-intensity continuous exercise: implications for exercise adherence. J Sports Sci 2011;29:547-553.

Blaak EE, Saris WH. Substrate oxidation, obesity and exercise training. Best Pract Res Clin Endocrinol Metab 2002;16:667-678.

Buemann B, Astrup A, Christensen NJ. Three months aerobic training fails to affect 24-hour energy expenditure in weight-stable, post-obese women. Int J Obes 1992;16:809-816.

Cohen J. Statistical power analysis for the behavioral sciences. New York (NY): Routledge Academic; 1988.

da Rocha EE, Alves VG, da Fonseca RB. Indirect calorimetry: methodology, instruments and clinical application. Curr Opin Clin Nutr Metab Care 2006;9:247-256.

DeLany JP, Windhauser MM, Champagne CM, Bray GA. Differential oxidation of individual dietary fatty acids in humans. Am J Clin Nutr 2000;72:905-911.

Fisher G, Hyatt TC, Hunter GR, Oster RA, Desmond RA, Gower BA. Effect of diet with and without exercise training on markers of inflammation and fat distribution in overweight women. Obesity (Silver Spring) 2011;19:1131-1136.

Friedewald WT, Levy RI, Fredrickson DS. Estimation of the concentration of low-density lipoprotein cholesterol in plasma, without use of the preparative ultracentrifuge. Clin Chem 1972;18:499-502.

Fujibayashi M, Hamada T, Matsumoto T, Kiyohara N, Tanaka S, Kotani K, Egawa K, Kitagawa Y, Kiso Y, Sakane N, Moritani T. Thermoregulatory sympathetic nervous system activity and diet-induced waist-circumference reduction in obese Japanese women. Am J Hum Biol 2009; 21:828-835.

Gao Y, Wang D, Xu ML, Shi SS, Xiong JF. Toxicological characteristics of edible insects in China: A historical review. Food Chem Toxicol 2018; 119:237-251
Gibala MJ, Little JP, Macdonald MJ, Hawley JA. Physiological adaptations to low-volume, high-intensity interval training in health and disease. J Physiol 2012;590:1077-1084.

Goodpaster BH, Katsiaras A, Kelley DE. Enhanced fat oxidation through physical activity is associated with improvements in insulin sensitivity in obesity. Diabetes 2003;52:2191-2197.

Jones PJ, Jew S, AbuMweis S. The effect of dietary oleic, linoleic, and linolenic acids on fat oxidation and energy expenditure in healthy men. Metabolism 2008;57:1198-1203.

Kang J, Ratamess N. Which comes first? Resistance before aerobic exercise or vice versa? ACSMs Health Fit J 2014;18:9-14.

Kien CL, Bunn JY, Ugrasbul F. Increasing dietary palmitic acid decreases fat oxidation and daily energy expenditure. Am J Clin Nutr 2005;82 :320-326.

Kim Y, Park J, Zhoh C, Hong S, Ryu K. The component analysis of male silkworm's extract. J Korean Soc Esthet Cosme 2010;5:37-45.

Kirk EP, Donnelly JE, Smith BK, Honas J, Lecheminant JD, Bailey BW, Jacobsen DJ, Washburn RA. Minimal resistance training improves daily energy expenditure and fat oxidation. Med Sci Sports Exerc 2009;41: $1122-1129$.

Kliewer SA, Sundseth SS, Jones SA, Brown PJ, Wisely GB, Koble CS, Devchand P, Wahli W, Willson TM, Lenhard JM, Lehmann JM. Fatty acids and eicosanoids regulate gene expression through direct interactions with peroxisome proliferator-activated receptors alpha and gamma. Proc Natl Acad Sci U S A 1997;94:4318-4323.

Krishnan S, Cooper JA. Effect of dietary fatty acid composition on substrate utilization and body weight maintenance in humans. Eur J Nutr 2014; 53:691-710.

Lavie CJ, Milani RV, Ventura HO. Obesity and cardiovascular disease: risk factor, paradox, and impact of weight loss. J Am Coll Cardiol 2009;53:1925-1932.

Longvah T, Manghtya K, Qadri SS. Eri silkworm: a source of edible oil with a high content of $\alpha$-linolenic acid and of significant nutritional value. J Sci Food Agric 2012;92:1988-1993.

Matthews DR, Hosker JP, Rudenski AS, Naylor BA, Treacher DF, Turner RC. Homeostasis model assessment: insulin resistance and beta-cell function from fasting plasma glucose and insulin concentrations in man. Diabetologia 1985;28:412-419.

Messina G, Valenzano A, Moscatelli F, Salerno M, Lonigro A, Esposito T, Monda V, Corso G, Messina A, Viggiano A, Triggiani AI, Chieffi S, Guglielmi G, Monda M, Cibelli G. Role of autonomic nervous system and orexinergic system on adipose tissue. Front Physiol 2017;8:137.

Millis RM, Austin RE, Hatcher MD, Bond V, Goring KL. Metabolic energy correlates of heart rate variability spectral power associated with a 900-calorie challenge. J Nutr Metab 2011;2011:715361. 
Nagai N, Sakane N, Moritani T. Metabolic responses to high-fat or low-fat meals and association with sympathetic nervous system activity in healthy young men. J Nutr Sci Vitaminol (Tokyo) 2005;51:355-360.

Nicklas BJ, Rogus EM, Goldberg AP. Exercise blunts declines in lipolysis and fat oxidation after dietary-induced weight loss in obese older women. Am J Physiol 1997;273(1Pt1):E149-E155.

Ortega JF, Morales-Palomo F, Fernandez-Elias V, Hamouti N, Bernardo FJ, Martin-Doimeadios RC, Nelson RK, Horowitz JF, Mora-Rodriguez R. Dietary supplementation with omega-3 fatty acids and oleate enhances exercise training effects in patients with metabolic syndrome. Obesity (Silver Spring) 2016;24:1704-1711.

Peronnet F, Massicotte D. Table of nonprotein respiratory quotient: an update. Can J Sport Sci 1991;16:23-29.

Piers LS, Walker KZ, Stoney RM, Soares MJ, O'Dea K. The influence of the type of dietary fat on postprandial fat oxidation rates: monounsaturated (olive oil) vs saturated fat (cream). Int J Obes Relat Metab Disord 2002;26:814-821.

Polley KR, Miller MK, Johnson M, Vaughan R, Paton CM, Cooper JA. Metabolic responses to high-fat diets rich in MUFA v. PUFA. Br J Nutr 2018;120:13-22.

Potteiger JA, Kirk EP, Jacobsen DJ, Donnelly JE. Changes in resting metabolic rate and substrate oxidation after 16 months of exercise training in overweight adults. Int J Sport Nutr Exerc Metab 2008;18:79-95.

Robertson MD, Jackson KG, Fielding BA, Morgan LM, Williams CM, Frayn $\mathrm{KN}$. Acute ingestion of a meal rich in $\mathrm{n}-3$ polyunsaturated fatty acids results in rapid gastric emptying in humans. Am J Clin Nutr 2002;76: 232-238.

Rosenkilde M, Nordby P, Nielsen LB, Stallknecht BM, Helge JW. Fat oxidation at rest predicts peak fat oxidation during exercise and metabolic phenotype in overweight men. Int J Obes (Lond) 2010;34:871-877.

Ryan D, Heaner M. Guidelines (2013) for managing overweight and obesity in adults. Preface to the full report. Obesity (Silver Spring) 2014; 22 Suppl 2:S1-S3.

Saini RK, Keum YS. Omega-3 and omega-6 polyunsaturated fatty acids: Dietary sources, metabolism, and significance - a review. Life Sci 2018; 203:255-267.

Schrauwen P, van Aggel-Leijssen DP, Hul G, Wagenmakers AJM, Vidal $\mathrm{H}$, Saris WHM, van Baak MA. The effect of a 3-month low-intensity endurance training program on fat oxidation and acetyl-CoA carboxylase-2 expression. Diabetes 2002;51:2220-2226.
Schutz Y, Tremblay A, Weinsier RL, Nelson KM. Role of fat oxidation in the long-term stabilization of body weight in obese women. Am J Clin Nutr 1992;55:670-674.

Schwingshackl L, Dias S, Strasser B, Hoffmann G. Impact of different training modalities on anthropometric and metabolic characteristics in overweight/obese subjects: a systematic review and network meta-analysis. PLoS One 2013;8:e82853.

Shanker KS, Shireesha K, Kanjilal S, Kumar S, Srinivas C, Rao J, Prasad R. Isolation and characterization of neutral lipids of desilked eri silkworm pupae grown on castor and tapioca leaves. J Agric Food Chem 2006; 54:3305-3309.

Sigal RJ, Alberga AS, Goldfield GS, Prud'homme D, Hadjiyannakis S, Gougeon R, Phillips P, Tulloch H, Malcolm J, Doucette S, Wells GA, Ma J, Kenny GP. Effects of aerobic training, resistance training, or both on percentage body fat and cardiometabolic risk markers in obese adolescents: the healthy eating aerobic and resistance training in youth randomized clinical trial. JAMA Pediatr 2014;168:1006-1014.

Solomon TP, Sistrun SN, Krishnan RK, Del Aguila LF, Marchetti CM, O'Carroll SM, O'Leary VB, Kirwan JP. Exercise and diet enhance fat oxidation and reduce insulin resistance in older obese adults. J Appl Physiol 2008;104:1313-1319.

Stevenson JL, Miller MK, Skillman HE, Paton CM, Cooper JA. A PUFArich diet improves fat oxidation following saturated fat-rich meal. Eur J Nutr 2017;56:1845-1857.

Tartibian B, Hajizadeh Maleki B, Kanaley J, Sadeghi K. Long-term aerobic exercise and omega-3 supplementation modulate osteoporosis through inflammatory mechanisms in post-menopausal women: a randomized, repeated measures study. Nutr Metab (Lond) 2011;8:71.

Villarroya F, Vidal-Puig A. Beyond the sympathetic tone: the new brown fat activators. Cell Metab 2013;17:638-643.

Weir JB. New methods for calculating metabolic rate with special reference to protein metabolism. J Physiol 1949;109:1-9.

Wilmore JH, Despres JP, Stanforth PR, Mandel S, Rice T, Gagnon J, Leon AS, Rao D, Skinner JS, Bouchard C. Alterations in body weight and composition consequent to $20 \mathrm{wk}$ of endurance training: the HERITAGE Family Study. Am J Clin Nutr 1999;70:346-352.

Yeo WK, Carey AL, Burke L, Spriet LL, Hawley JA. Fat adaptation in well-trained athletes: effects on cell metabolism. Appl Physiol Nutr Metab 2011;36:12-22 